\title{
Current transport mechanisms in plasma-enhanced atomic layer deposited AIN thin films
}

Halit Altuntas, Cagla Ozgit-Akgun, Inci Donmez, and Necmi Biyikli

Citation: Journal of Applied Physics 117, 155101 (2015);

View online: https://doi.org/10.1063/1.4917567

View Table of Contents: http://aip.scitation.org/toc/jap/117/15

Published by the American Institute of Physics

\section{Articles you may be interested in}

Epitaxial growth of AIN films via plasma-assisted atomic layer epitaxy

Applied Physics Letters 103, 082110 (2013); 10.1063/1.4818792

Atomic layer deposition of $\mathrm{GaN}$ at low temperatures

Journal of Vacuum Science \& Technology A: Vacuum, Surfaces, and Films 30, 01A124 (2011);

$10.1116 / 1.3664102$

Structural and chemical analysis of annealed plasma-enhanced atomic layer deposition aluminum nitride films Journal of Vacuum Science \& Technology A: Vacuum, Surfaces, and Films 34, 041506 (2016);

$10.1116 / 1.4953029$

Optical properties of AIN thin films grown by plasma enhanced atomic layer deposition Journal of Vacuum Science \& Technology A: Vacuum, Surfaces, and Films 30, 021506 (2012); $10.1116 / 1.3687937$

Low-temperature self-limiting atomic layer deposition of wurtzite $\operatorname{lnN}$ on $\mathrm{Si}(100)$

AIP Advances 6, 045203 (2016); 10.1063/1.4946786

Crystal AIN deposited at low temperature by magnetic field enhanced plasma assisted atomic layer deposition Journal of Vacuum Science \& Technology A: Vacuum, Surfaces, and Films 31, 01 A114 (2012);

$10.1116 / 1.4764112$

\section{Scilight}

Sharp, quick summaries illuminating the latest physics research

\section{Sign up for FREE!}

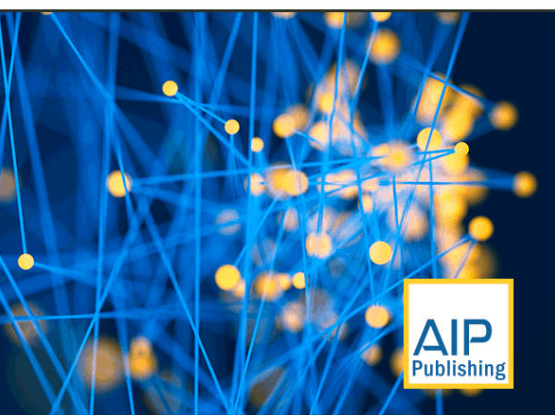




\title{
Current transport mechanisms in plasma-enhanced atomic layer deposited AIN thin films
}

\author{
Halit Altuntas, ${ }^{1, a)}$ Cagla Ozgit-Akgun, ${ }^{2,3}$ Inci Donmez, ${ }^{2,3}$ and Necmi Biyikli ${ }^{2,3, a)}$ \\ ${ }^{1}$ Faculty of Science, Department of Physics, Cankiri Karatekin University, Cankiri 18100, Turkey \\ ${ }^{2}$ National Nanotechnology Research Center (UNAM), Bilkent University, Bilkent, Ankara 06800, Turkey \\ ${ }^{3}$ Institute of Materials Science and Nanotechnology, Bilkent University, Bilkent, Ankara 06800, Turkey
}

(Received 4 February 2015; accepted 2 April 2015; published online 15 April 2015)

\begin{abstract}
Here, we report on the current transport mechanisms in AlN thin films deposited at a low temperature (i.e., $200^{\circ} \mathrm{C}$ ) on $p$-type $\mathrm{Si}$ substrates by plasma-enhanced atomic layer deposition. Structural characterization of the deposited AlN was carried out using grazing-incidence X-ray diffraction, revealing polycrystalline films with a wurtzite (hexagonal) structure. Al/AlN/ $p$-Si metal-insulatorsemiconductor (MIS) capacitor structures were fabricated and investigated under negative bias by performing current-voltage measurements. As a function of the applied electric field, different types of current transport mechanisms were observed; i.e., ohmic conduction (15.2-21.5 MV/m), Schottky emission (23.6-39.5 MV/m), Frenkel-Poole emission (63.8-211.8 MV/m), trap-assisted tunneling (226-280 MV/m), and Fowler-Nordheim tunneling (290-447 MV/m). Electrical properties of the insulating AlN layer and the fabricated $\mathrm{Al} / \mathrm{AlN} / p$-Si MIS capacitor structure such as dielectric constant, flat-band voltage, effective charge density, and threshold voltage were also determined from the capacitance-voltage measurements. (C) 2015 AIP Publishing LLC.
\end{abstract}

[http://dx.doi.org/10.1063/1.4917567]

\section{INTRODUCTION}

Binary and ternary III-nitride semiconductors are appealing materials for the micro- and opto-electronics industries due to their potential device applications. ${ }^{1-4}$ Among this group of materials, aluminum nitride (AlN) attracted lots of attention due to its direct and wide band gap of $6.2 \mathrm{eV}$, excellent thermal conductivity, good piezoelectric properties, good chemical and thermal stability, and high acoustic velocity. Moreover, AlN has a high dielectric constant, which makes it a potential insulating material for metal-insulator-semiconductor (MIS) devices. ${ }^{5}$ AlN thin films have been deposited by various methods including molecular beam epitaxy, ${ }^{6}$ metalorganic chemical vapor deposition (MOCVD), ${ }^{7}$ ion beam deposition, ${ }^{8}$ magnetron sputtering, ${ }^{9}$ pulsed laser deposition, ${ }^{10}$ and plasma-enhanced chemical vapor deposition. ${ }^{11}$ Although MOCVD-grown AIN thin films are being widely used in today's semiconductor technology, MOCVD growth inherently takes place at high temperatures, which is incompatible with device applications requiring the use of temperature sensitive device layers and flexible substrates. When considered from this point of view, atomic layer deposition (ALD) is an alternative low-temperature thin film deposition method, which consists of sequential and non-overlapping injections of two or more gas-phase precursors with a well-defined periodicity. The ALD technique is inherently self-limiting; once a monomolecular layer of a precursor is chemisorbed on the substrate surface, the gas-solid reaction stops since the precursor molecules of the same kind do not react with each other. The critical advantages of ALD over other deposition techniques can therefore be listed as the deposition of

\footnotetext{
a) Authors to whom correspondence should be addressed. Electronic addresses: altunhalit@gmail.com and biyikli@unam.bilkent.edu.tr.
}

highly conformal, uniform, and smooth thin films with subnanometer thickness control, deposition at low temperatures, and low residual stress in the deposited layers. ${ }^{12,13}$ ALD temperatures can also be further decreased by the enhancement of surface reactions via an additional energy source, such as plasma. Plasma-enhanced ALD (PEALD) is a widely used technique, in which plasma source creates highly reactive radicals that contribute to chemical reactions occurring at the substrate surface. When compared to conventional thermal ALD, PEALD provides a wider range of materials deposition capability at relatively low substrate temperatures.

In the previous reports, we investigated the self-limiting growth of crystalline AlN thin films at low temperatures by PEALD using trimethylaluminum (TMA) and ammonia $\left(\mathrm{NH}_{3}\right)$ as the aluminum and nitrogen precursors, respectively. ${ }^{14,15}$ Here, we present the room-temperature electrical conduction properties of these polycrystalline wurtzite AlN thin films, along with some other electrical properties, as determined from the current-voltage $(I-V)$ and high-frequency $(1 \mathrm{MHz})$ capacitancevoltage $(C-V)$ measurements of $\mathrm{Al} / \mathrm{AlN} / p$-Si MIS structures.

\section{EXPERIMENTAL METHOD}

MIS capacitor structures with AlN as the insulating layer were fabricated on $25 \mathrm{~mm} \times 40 \mathrm{~mm} p$-type $\mathrm{Si}$ (100) substrates $(\rho=20-40 \Omega \mathrm{cm})$ using class 100 and 1000 cleanroom facilities. Solvent-cleaned substrates were subjected to piranha etch $\left(\mathrm{H}_{2} \mathrm{SO}_{4}: \mathrm{H}_{2} \mathrm{O}_{2}=4: 1\right)$ for 5-10 min, which was followed by the native oxide removal in dilute hydrofluoric acid solution (HF, 2 vol. \%) for 2-3 min. Back ohmic contacts were formed by thermal evaporation and subsequent rapid thermal annealing. First, $\sim 80 \mathrm{~nm}$ thick $\mathrm{Al}$ was deposited to the back side of each wafer using VAKSIS Thermal Evaporation System (PVD Vapor 3S Thermal), while the top 
sides were protected with a layer of photoresist. After stripping the photoresist layers and washing the samples alternately with acetone, methanol, isopropanol, and deionized (DI) water, and then drying with $\mathrm{N}_{2}$, samples were annealed in ATV-Unitherm (RTA SRO-704) rapid thermal annealing system at $450{ }^{\circ} \mathrm{C}$ for $2 \mathrm{~min}$ under $100 \mathrm{sccm} \mathrm{N}_{2}$ flow. Ramping rate was $15^{\circ} \mathrm{C} / \mathrm{min}$. Si (100) substrates with back ohmic contacts and solvent-cleaned bare Si (100) substrates (for characterization purposes) were then loaded into a Fiji F200-LL ALD reactor (Cambridge Nanotech Inc.) immediately after they were dipped into dilute HF solution, rinsed with DI water and dried with $\mathrm{N}_{2} .175$ cycles AlN were deposited on these substrates at $200^{\circ} \mathrm{C}$, where one PEALD cycle consisted of: $0.1 \mathrm{~s}$ TMA pulse $/ 10 \mathrm{~s}$ Ar purge $/ 40 \mathrm{~s}$, $50 \mathrm{sccm}, 300 \mathrm{~W} \mathrm{NH}_{3}$ plasma/10 s Ar purge. During the deposition, TMA and $\mathrm{NH}_{3}$ were carried from separate lines using 60 and $200 \mathrm{sccm} \mathrm{Ar}$, respectively. Base pressure was $30 \mathrm{~Pa}$. Top contacts were then formed by thermal evaporation $(\sim 80 \mathrm{~nm}$ thick $\mathrm{Al})$ and photolithography. $\mathrm{Al}$ and $\mathrm{AlN}$ layers were patterned simultaneously to obtain $250 \mu \mathrm{m} \times 250 \mu \mathrm{m}$ MIS devices during the development of AZ $5214 \mathrm{E}$ photoresist with $\mathrm{AZ} 400 \mathrm{~K}$ developer ( $\mathrm{AZ} 400 \mathrm{~K}: \mathrm{H}_{2} \mathrm{O}=1: 4$ ).

Capacitance-voltage $(C-V)$ and current-voltage $(I-V)$ characteristics of the fabricated MIS capacitor structures were measured under dark using a semiconductor parameter analyzer (Keithley 4200-SCS), which is connected to a probe station (Cascade Microtech PM-5). $C$ - $V$ curves were obtained at a frequency of $1 \mathrm{MHz}$. AlN films deposited on solvent-cleaned and HF-dipped bare Si (100) substrates were characterized by spectroscopic ellipsometry, X-ray photoelectron spectroscopy (XPS), X-ray reflectivity (XRR), and grazing incidence $\mathrm{X}$-ray diffraction (GIXRD). Ellipsometric spectra of the films were recorded in the wavelength range of $300-850 \mathrm{~nm}$ at three angles of incidence $\left(65^{\circ}, 70^{\circ}\right.$, and $\left.75^{\circ}\right)$ using a variable angle spectroscopic ellipsometer (J.A. Woollam). Optical constants and film thicknesses were extracted using the Cauchy dispersion function using a twolayer model; i.e., Cauchy/Si $(0.5 \mathrm{~mm})$. Chemical compositions of the films were determined by XPS using Thermo Scientific K-Alpha spectrometer with a monochromatized Al $\mathrm{K} \alpha \mathrm{X}$-ray source. The pass energy, step size, and spot size were $30 \mathrm{eV}, 0.1 \mathrm{eV}$, and $400 \mathrm{~mm}$, respectively. Etching of the samples was carried out in situ with a beam of Ar ions having an acceleration voltage of $1 \mathrm{kV}$. XRR and GIXRD measurements were performed in a PANalytical X'Pert PRO MRD diffractometer using $\mathrm{Cu} \mathrm{K} \alpha$ radiation.

\section{RESULTS AND DISCUSSION}

\section{A. Film structure and capacitance-voltage (C-V) characteristics}

GIXRD pattern of the AlN thin film deposited at $200^{\circ} \mathrm{C}$ on a $\mathrm{Si}(100)$ substrate by the application of 175 PEALD cycles is given in Fig. 1. On the pattern, (100), (002), (101), (102), (110), (103), and (112) reflections of the hexagonal phase were observed (ICDD reference code: 00-025-1133), indicating a polycrystalline AlN film with wurtzite (hexagonal) structure. The refractive index $(n)$ of the PEALD-grown AlN thin film was measured within the wavelength range of

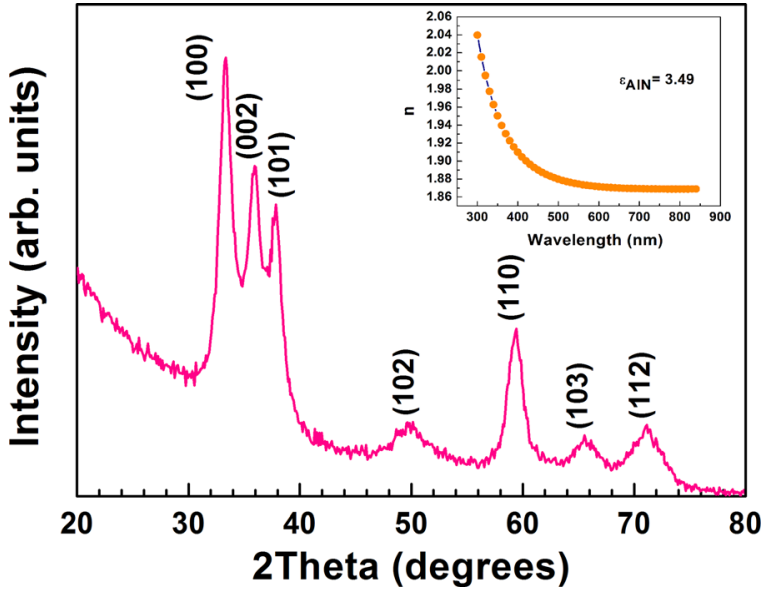

FIG. 1. GIXRD pattern of the AlN thin film deposited at $200^{\circ} \mathrm{C}$ on $\mathrm{Si}$ (100) substrate. Film is polycrystalline with a hexagonal wurtzite structure. Inset shows the spectral refractive index $(n)$ of the deposited AlN thin film.

$300-850 \mathrm{~nm}$ by spectroscopic ellipsometry. As can be seen from the inset of Fig. 1, refractive index decreases from 2.04 to 1.87 as the wavelength increases from $300 \mathrm{~nm}$ to $850 \mathrm{~nm}$. As known, $n$ value depends on film crystallinity; the $n$ values that were reported in the literature for polycrystalline AIN films ${ }^{16}$ are in good agreement with the present data, which again confirms the polycrystalline nature of the deposited films. The optical dielectric constant $\left(\kappa_{\mathrm{op}}\right)$ is equal to the square of the refractive index, which was already obtained by spectroscopic ellipsometry (i.e., $\kappa_{\mathrm{op}}=n^{2}$ ). $n$ value of the deposited AlN was measured to be 1.87 at $633 \mathrm{~nm}$, resulting in a $\kappa_{\text {op }}$ of 3.49 .

The typical high-frequency ( $1 \mathrm{MHz}) C-V$ characteristics of $\mathrm{Al} / \mathrm{AlN} / p$-Si MIS capacitors are presented in Fig. 2. The $C-V$ characteristics exhibited a negative flat-band voltage shift, which is associated with the presence of trapped positive charges as the nitride equivalent of fixed oxide charges in the dielectric films or AlN/Si interface. Al/AlN/p-Si MIS capacitors also showed $C$ - $V$ hysteresis behavior (see inset of Fig. 2) due to the presence of mobile charges inside the AlN film and trapped charges at the interface. The $C$ - $V$ curve looped counterclockwise when swept from accumulation to

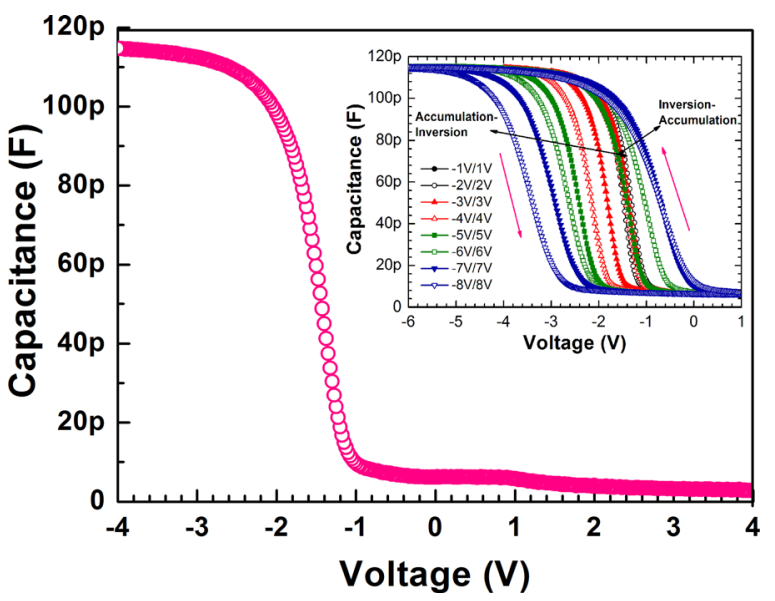

FIG. 2. High-frequency $C$ - $V$ curve of an Al/AlN $/ p$-Si MIS device fabricated using PEALD-grown AIN insulating layer. Inset shows the hysteresis behaviors of the fabricated MIS capacitors. 
inversion, implying that the hysteresis behavior is mainly due to hole injection from $\mathrm{Si}$ into the dielectric AIN layer since flat-band voltage shifts in the accumulation-inversion branches are larger than those in the inversion-accumulation branches. The magnitude of the hysteresis width (i.e., memory window) was found to be dependent on the voltage sweeping range. In general, the memory window increases with gate bias, indicating the injection of increased number of charges. ${ }^{17}$

The effective charges - depending on their type-shift the $C$ - $V$ curve to either left or right. The effective charge density $\left(N_{e f f}\right)$ can be calculated using the flat-band voltage $\left(V_{F B}\right)$ that is graphically determined from the $C-V$ curves. The $N_{\text {eff }}$ and flat-band voltage are related to each other by

$$
N_{e f f}=\frac{C_{i}\left(\phi_{m s}-V_{F B}\right)}{q A},
$$

where $\phi_{m s}$ is the difference between metal (Al) and semiconductor $(\mathrm{Si})$ work functions, $q$ is the electronic charge, $C_{i}$ is the capacitance of the insulator under strong accumulation mode, and $A$ is the area of the capacitor, which was calculated to be $5.93 \times 10^{-4} \mathrm{~cm}^{2}$ for the present case.

Electrical and optical properties of the materials are influenced by the dielectric constant $\left(\varepsilon_{r}\right)$, which can be obtained by substituting the value of $C_{i}$ under strong accumulation mode into the following equation:

$$
C_{i}=\frac{\varepsilon_{r} \varepsilon_{o} A}{t_{d}}
$$

where $\varepsilon_{o}$ is the permittivity of vacuum and $t_{d}$ is the thickness of the dielectric layer (i.e., $\sim 14.4 \mathrm{~nm}$ ). The dielectric constant was therefore estimated to be 3.15 for the deposited AlN film. In the following text, this dielectric constant will be mentioned as the static dielectric constant.

In order to determine the flat-band voltage, flat-band capacitance $\left(C_{F B}\right)$ method was used. The $p$-type Si surface at the flat-band condition is given by

$$
C_{F B S}=\frac{\varepsilon_{S i} \varepsilon_{o}}{\lambda},
$$

where $\varepsilon_{\mathrm{Si}}$ is the dielectric constant of Si (i.e., 11.8) and $\lambda$ is the Debye length of $p$-type $\mathrm{Si}$, which is expressed as

$$
\lambda=\left(\frac{\varepsilon_{S i} \varepsilon_{o} k T}{q^{2} N_{A}}\right)^{1 / 2}
$$

where $k$ is the Boltzmann constant, $T$ is the absolute temperature, and $N_{A}$ is the doping concentration of $p$-type Si. The $N_{A}$ values were calculated from the slopes of $1 / C^{2}-V$ graphs and found to be $1.13 \times 10^{15} \mathrm{~cm}^{-3}$. Following the calculation of $C_{F B S}$, the flat-band capacitance $C_{F B}$ can be obtained using the series capacitance relationship

$$
C_{F B}=\frac{C_{o} C_{F B S}}{C_{o}+C_{F B S}} .
$$

The flat-band voltage $\left(V_{F B}\right)$ is the voltage value corresponding to $C_{F B}$ on the high-frequency $C-V$ curves, which was graphically determined to be $-1.35 \mathrm{~V}$. By substituting this value in Eq. (1), $N_{\text {eff }}$ was found as $6.4 \times 10^{11} \mathrm{~cm}^{-2}$ for the deposited films. Calculated electrical parameters of PEALD-grown AIN thin films are summarized in Table I. Effective charge density $\left(N_{\text {eff }}\right)$ values on the order of $10^{12} \mathrm{~cm}^{-2}$ and $10^{13} \mathrm{~cm}^{-2}$ were reported for AIN thin films deposited by sequential injection of TMA and $\mathrm{NH}_{3}$ under UV radiation, ${ }^{18}$ radio frequency (RF) magnetron sputtering, ${ }^{19}$ reactive sputtering, ${ }^{20}$ d.c. magnetron sputtering, ${ }^{21}$ and RF magnetron sputtering. ${ }^{22}$ The value reported herein (i.e., $6.4 \times 10^{11} \mathrm{~cm}^{-2}$ ) is therefore better than those reported in the literature for AIN thin films deposited using various methods and suggests that AIN thin films with low effective charge densities can be deposited successfully using low-temperature PEALD.

Another important electrical parameter of a capacitor is the threshold onset voltage $\left(V_{t h}\right)$. This parameter can be calculated for strong inversion of the MIS device ( $p$-type substrate) according to the following relation: ${ }^{23}$

$$
V_{t h}=V_{F B}+2 \phi_{b}+\frac{\left(4 q \varepsilon_{S i} \varepsilon_{o} N_{A}\left|\phi_{b}\right|\right)^{1 / 2}}{\varepsilon_{o x} \varepsilon_{o} / t_{o x}},
$$

where $\phi_{b}=\left(k_{B} T / q\right) \ln \left(N_{A} / n_{i}\right)$ and $n_{i}$ is the intrinsic carrier concentration of $\mathrm{Si}$ at room temperature (i.e., $1.45 \times 10^{10} \mathrm{~cm}^{-3}$ ). Substituting these parameters into Eq. (6) yields the $V_{t h}$ value as $-0.67 \mathrm{~V}$ for $\mathrm{Al} / \mathrm{AlN} / p$-Si MIS capacitors investigated in this study.

\section{B. Current transport mechanisms under negative bias}

Electrical conduction behaviors of the AlN capacitors were investigated under d.c. bias. Measured current densities $(J)$ of the $\mathrm{Al} / \mathrm{AlN} / p$-Si capacitors under negative gate bias (NGB) are given in Fig. 3. It is well-known that many conduction mechanisms can be observed for dielectric materials depending on the magnitude of applied electric field. For AlN thin films deposited using PEALD, we observed five different regions on the $J-V$ plot, which represent different transport mechanisms under NGB. Each of these regions will be explained in detail in the following paragraphs along with the corresponding current transport mechanisms.

Fig. 4 shows the $\ln (J)$ vs. In $(E)$ graph for $\mathrm{Al} / \mathrm{AlN} / p-\mathrm{Si}$ capacitors under NGB, where $E$ is the electric field, values of which were obtained from the formula $E=\left(V-V_{F B}\right) / t_{A I N}$. As seen from this figure, plot is initially linear at the low electric field region (i.e., 15.2-21.5 MV/m). In this region, current density is proportional to the electric field, and the slope of the plot is about 1 . This behavior indicates that the

TABLE I. Electrical parameters of the PEALD-grown AlN thin films as extracted from high-frequency $C$ - $V$ curves.

\begin{tabular}{lcccccc}
\hline \hline$\lambda$-Debye length $(\mathrm{cm})$ & $C_{\mathrm{FBS}}(\mathrm{F})$ & $C_{\mathrm{i}}(\mathrm{F})$ & $C_{\mathrm{FB}}(\mathrm{F})$ & $V_{\mathrm{FB}}(\mathrm{V})$ & $\phi_{m s}(\mathrm{eV})$ & $\varepsilon_{r}$ \\
\hline $1.2 \times 10^{-5}$ & $5.08 \times 10^{-11}$ & $1.15 \times 10^{-10}$ & $3.52 \times 10^{-11}$ & -1.35 & -0.82 & 3.15 \\
\hline \hline
\end{tabular}




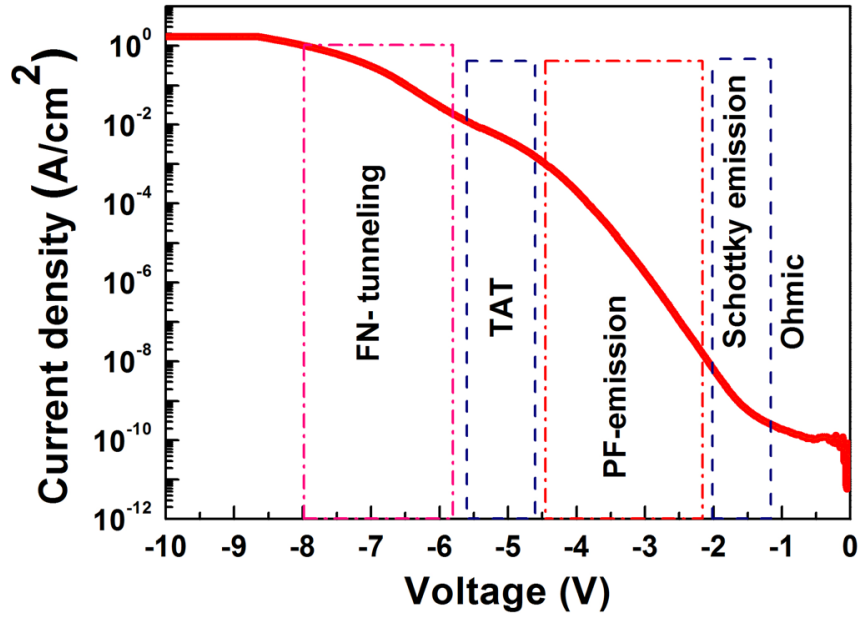

FIG. 3. Current density-voltage characteristics of the $\mathrm{Al} / \mathrm{AlN} / p$-Si MIS capacitor structures.

current transport mechanism in this $E$ range is Ohmic conduction.

The Schottky emission (SE) mechanism is described as

$$
J \propto A^{*} T^{2} \exp \left[\frac{-q\left(\phi_{B}-\sqrt{q E / 4 \pi \varepsilon_{r} \varepsilon_{o}}\right)}{k_{B} T}\right],
$$

where $J$ is the current density, $E$ is the electric field, $A^{*}$ is the effective Richardson constant, $\phi_{B}$ is the barrier height, $\varepsilon_{r}$ is the dielectric constant, $q$ is the electronic charge, and $k$ is Boltzmann constant. If SE applies, then the $\varepsilon_{r}$ value can be calculated from the $\ln (J)$ vs. $E^{1 / 2}$ plot using Eq. (7). From the $\ln (J)$ vs. $E^{1 / 2}$ graph (see Fig. 5), $\phi_{B}$ and $\varepsilon_{r}$ were calculated to be $1.02 \mathrm{eV}$ and 3.17 , respectively. The calculated $\varepsilon_{r}$ value is in good agreement with the values obtained from high frequency $C-V$ measurement (i.e., 3.15) and spectroscopic ellipsometry $\left(\kappa_{\mathrm{op}}=3.49\right)$. These results indicate that the conduction mechanism in the $E$ range of $23.6-39.5 \mathrm{MV} / \mathrm{m}$ is SE.

Current conduction in PEALD-grown AlN films is governed by a different transport mechanism, FrenkelPoole emission $(F P)$, under the substrate hole injection and at relatively high electric fields (i.e., 63.8-211.8 MV/m).

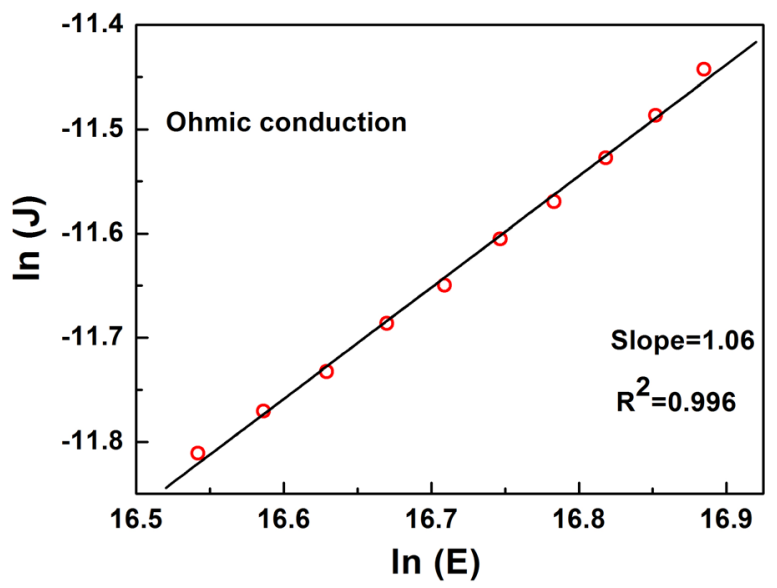

FIG. 4. $\ln (J)$ vs. $\ln (E)$ plot of the Al/AlN/p-Si MIS capacitor structures.

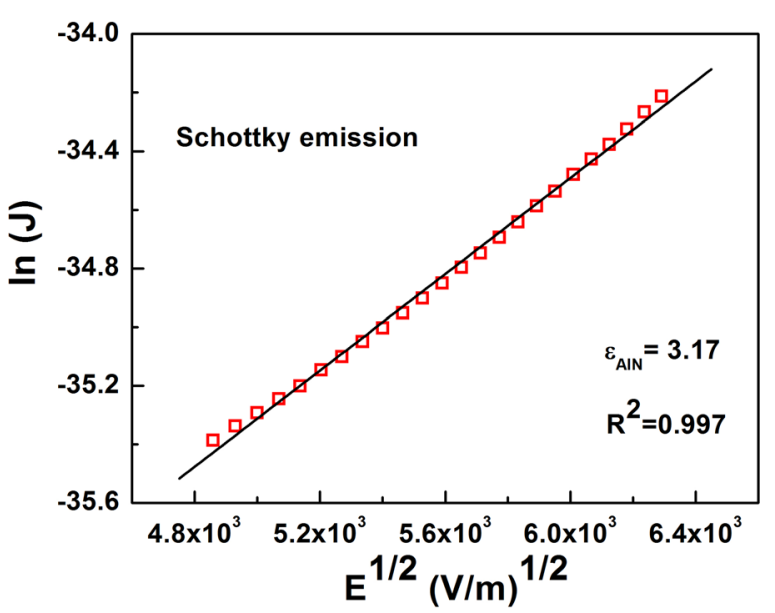

FIG. 5. Schottky emission model fitted for the determination of conduction mechanisms in $\mathrm{Al} / \mathrm{AlN} / p$-Si MIS capacitor structures.

FP is the field-assisted thermal de-trapping of a carrier from bulk dielectric into conduction band; therefore, it is a "bulk-limited" conduction process. Current density due to FP is given as

$$
J \propto E \exp \left[\frac{-q\left(\phi_{t}-\sqrt{q E / \pi \varepsilon_{r} \varepsilon_{o}}\right)}{k_{B} T}\right],
$$

where $\phi_{t}$ is the trap energy level; i.e., the energy level of the electrically active defect states within the band gap of a dielectric material. According to Eq. (8), the slope of the $\ln$ $(J / E)$ vs. $E^{1 / 2}$ plot provides an estimate for the dielectric constant. The plot is very well fitted $\left(\mathrm{R}^{2}=0.999\right)$ with the FP mechanism as shown in Fig. 6, and $\phi_{t}$ and $\varepsilon_{r}$ were calculated as $0.98 \mathrm{eV}$ and 3.82 , respectively. This $\varepsilon_{r}$ value agrees well with the optical and static dielectric constants, thus confirming the presence of FP conduction mechanism in the 63.8-211.8 MV/m range. The $\phi_{t}$ value calculated in this study (i.e., $0.98 \mathrm{eV}$ ) is higher than those reported by Engelmark et al. ${ }^{20}$ and Hassine et al. $^{24}$ According to $\mathrm{Wu}$ et al., ${ }^{25}$ dangling bonds in the aluminum tend to give rise to $\sim 1 \mathrm{eV}$ in $\phi_{t}$ for metal-rich AlN films. XPS analysis of the PEALD-grown film revealed $\mathrm{Al} / \mathrm{N}$ ratios of 1.5 and 1.03 at the film surface and bulk, respectively, indicating slightly Al-rich AlN thin films. The higher value of the $\phi_{t}$ as

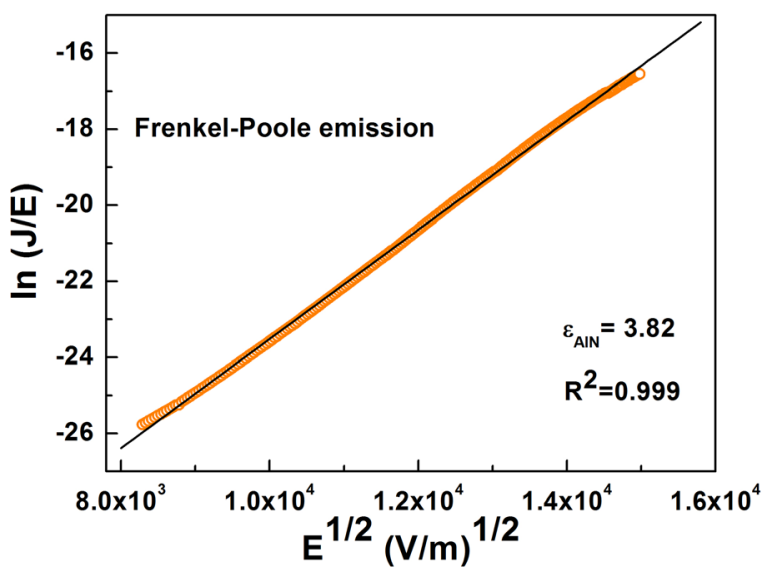

FIG. 6. Frenkel-Poole plot of the Al/AlN/p-Si MIS capacitor structures. 
compared to those reported in the literature might therefore be attributed to the dangling bonds of excess aluminum in deposited films.

Fig. 7 is the $\ln (J)$ vs. $1 / E$ under the substrate injection condition. At moderately high electric fields (i.e., 226-280 $\mathrm{MV} / \mathrm{m})$, a perfect linear variation $\left(\mathrm{R}^{2}=1\right)$ of $\ln (J)$ vs. $1 / E$ was observed, which points out that the conduction mechanism within this range is trap assisted tunneling (TAT). The current due to TAT mechanism is given by the following equation:

$$
J_{T A T} \propto \exp \left\{\frac{-8 \pi \sqrt{2 q m_{A I N}}}{3 h E} \phi_{t}^{3 / 2}\right\}
$$

where $\phi_{t}$ is the energy level of the electronic defect states, which was graphically obtained from the plot of $\ln (J)$ vs. $1 / E$ as $0.6 \mathrm{eV}$. The obtained $\phi_{t}$ value is equal to that of a nitrogen vacancy in crystalline AIN as predicted by Jenkins and Dow. ${ }^{26}$ Moreover, Ligatchev et al. ${ }^{27}$ reported defect state energy levels of $0.47-0.62 \mathrm{eV}$ and $0.73-0.80 \mathrm{eV}$ for nitrogen vacancies in AlN deposited on silicon. Thus, the obtained energy level of electronic defect states is related to the nitrogen vacancies, which are responsible for electron conduction in AlN.

Finally, for the $290-447 \mathrm{MV} / \mathrm{m}$ electric field region in the $J-V$ plot, the current density was fitted by the FowlerNordheim (FN) tunneling mechanism. The FN tunneling current density is given as

$$
J_{F N} \propto E^{2} \exp \left\{\frac{-8 \pi \sqrt{2 q m_{A l N}}}{3 h E} \phi_{b}^{3 / 2}\right\},
$$

where $\phi_{b}$ is the barrier height at the emitting interface. Fig. 8 is the plot of $\ln \left(J / E^{2}\right)$ vs. $1 / E$, which shows a linear relationship for the indicated electric field range. From the slope of this plot, $\phi_{b}$ was calculated to be $0.84 \mathrm{eV}$. However, the derived barrier height is much lower than $3.48 \mathrm{eV}$ at the $\mathrm{Al} / \mathrm{AlN}$ interface. Therefore, an electron transferring from metal gate to the dielectric may be emitted from electronic defect states at the metal/dielectric interface via FN tunneling.

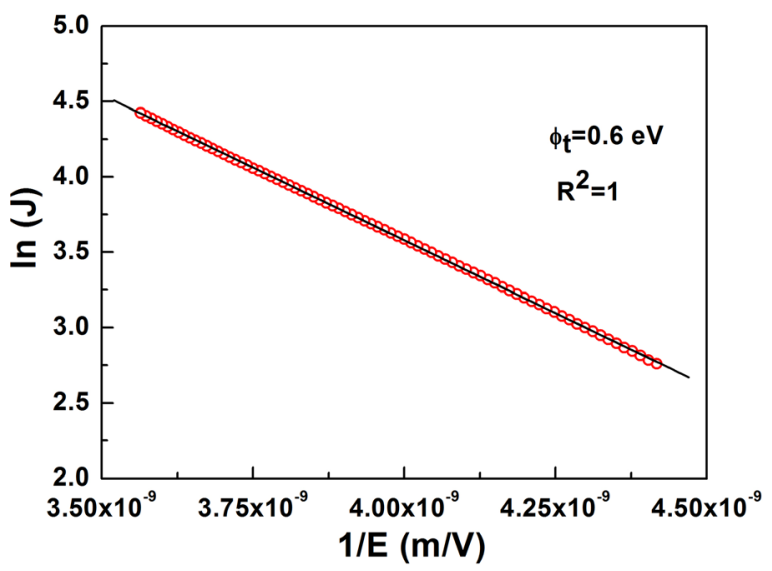

FIG. 7. $\ln (J)$ vs. $1 / E$ plot (i.e., the TAT plot).

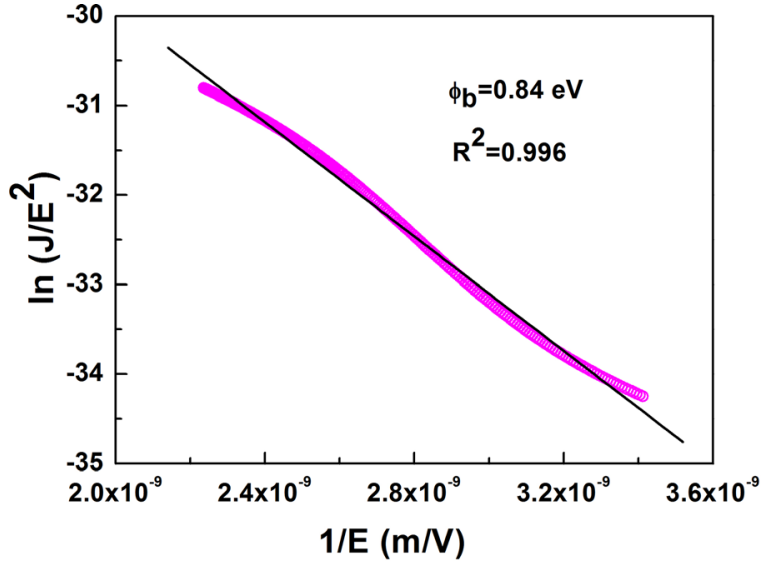

FIG. $8 . \ln \left(J / E^{2}\right)$ vs. 1/E plot (i.e., the FN tunneling plot).

\section{CONCLUSION}

In this work, polycrystalline wurtzite AlN thin films were deposited on $p$-Si substrates at $200^{\circ} \mathrm{C}$ by PEALD, using TMA and $\mathrm{NH}_{3}$ as the aluminum and nitrogen precursors, respectively. Electric-field dependent current transport mechanisms of the fabricated $\mathrm{Al} / \mathrm{AlN} / p-\mathrm{Si}$ MIS structures were investigated. Within the low-field regime, the conduction mechanisms were determined as ohmic conduction and Schottky emission (15.2-21.5 MV/m and 23.6-39.5 MV/m, respectively), whereas for higher electric field, the conduction mechanisms were found to be Frenkel-Poole emission (63.8-211.8 MV/m), trap assisted tunneling (226-280 MV/m), and Fowler-Nordheim tunneling (290-447 MV/m). The calculated energy levels of the electronic defect states are attributed to the dangling bonds present as a result of excess aluminum, and nitrogen vacancies in the polycrystalline AIN. Furthermore, several critical electrical parameters of the capacitor structures, as well as the insulating layer, such as dielectric constant, flat-band voltage, effective charge density, and threshold voltage were determined using the $C-V$ measurements. The results show that these low-temperature PEALDgrown AIN films have a significant potential for various electronic applications. Recently, we deposited AlN thin films with reduced impurity contents using a novel approach, which relies on the use of a hollow cathode plasma source. Electrical properties of these higher quality PEALD-grown AIN thin films are still under investigation.

\section{ACKNOWLEDGMENTS}

This work was performed at UNAM supported by the State Planning Organization (DPT) of Turkey through the National Nanotechnology Research Center Project. N. Biyikli acknowledges Marie Curie International Reintegration Grant (IRG) for funding NEMSmart (PIRG05-GA-2009-249196) project.

${ }^{1}$ H. Oikawa, R. Akiyama, K. Kanazawa, S. Kuroda, I. Harayama, K. Nagashima, D. Sekiba, Y. Ashizawa, A. Tsukamoto, K. Nakagawa, and N. Ota, Thin Solid Films 574, 110 (2015).

${ }^{2}$ I. Yonenaga, Y. Ohno, T. Yao, and K. Edagawa, J. Cryst. Growth 403, 72 (2014).

${ }^{3}$ S. Nakamura, Jpn. J. Appl. Phys., Part 2 35, L74 (1996). 
${ }^{4}$ H. Morkoç, S. Strite, G. B. Gao, M. E. Lin, B. Sverdlov, and M. Burns, J. Appl. Phys. 76, 1363 (1994).

${ }^{5}$ H. Van Bui, M. D. Nguyen, F. B. Wiggers, A. A. I. Aarnink, M. P. de Joung, and A. Y. Kovalgin, ECS J. Solid State Sci. Technol. 3, P101 (2014). ${ }^{6}$ M. Placidi, A. Perez-Tomas, J. C. Moreno, E. Frayssinet, F. Semond, A. Constant, P. Godignon, N. Mestres, A. Crespi, and J. Millan, Surf. Sci. 604, 63 (2010).

${ }^{7}$ C. M. Zetterling, M. Oestling, C. I. Harris, N. Nordell, K. Wongchotigul, and M. G. Spencer, Mater. Sci. Forum 264, 877 (1998).

${ }^{8}$ C. Men and C. Lin, Mater. Sci. Eng., B 133, 124 (2006).

${ }^{9}$ M. A. Moreira, I. Doi, J. F. Souza, and J. A. Diniz, Microelectron. Eng. 88, 802 (2011).

${ }^{10}$ Y. E. Lu, Z. M. Ren, T. C. Chong, B. A. Cheong, S. K. Show, and J. P. Wang, J. Appl. Phys. 87, 1540 (2000).

${ }^{11}$ G. Sanchez, B. Abdallah, P. Tristant, C. Dublanche-Tixier, M. A. Djouadi, M. P. Besland, P. Y. Jouan, and A. B. Alles, J. Mater. Sci. 44, 6125 (2009).

${ }^{12}$ H. Kim, J. Vac. Sci. Technol., B 21, 2231 (2003).

${ }^{13}$ O. M. E. Ylivaara, X. Liu, L. Kilpi, J. Lyytinenn, D. Schneider, M. Laitinen, J. Julin, S. Ali, S. Sintonen, M. Berdova, E. Haimi, T. Sajavaara, H. Ronkaien, H. Lipsanen, J. Koskinen, S.-P. Hannula, and R. L. Puurunen, Thin Solid Films 552, 124 (2014).

${ }^{14}$ C. Ozgit, I. Donmez, M. Alevli, and N. Biyikli, Thin Solid Films 520, 2750 (2012).
${ }^{15}$ M. Alevli, C. Ozgit, I. Donmez, and N. Biyikli, Phys. Status Solidi A 209, 266 (2012).

${ }^{16}$ H. C. Barshilia, B. Deepthi, and K. S. Rajam, Thin Solid Films 516, 4168 (2008).

${ }^{17}$ W. Guan, S. Long, M. Liu, Z. Li, Y. Hu, and Q. Liu, J. Phys. D: Appl. Phys. 40, 2754 (2007).

${ }^{18}$ D. Eom, S. Y. No, C. S. Hwang, and H. J. Kim, J. Electrochem. Soc. 153, C229 (2006).

${ }^{19}$ K. Jang, K. Lee, J. Kim, S. Hwang, J. Lee, S. K. Dhungel, S. Jung, and J. Yi, Mater. Sci. Semicond. Process. 9, 1137 (2006).

${ }^{20}$ F. Engelmark, J. Westlinder, G. F. Iriarte, I. V. Katardjiev, and J. Olsson, IEEE Trans. Electron Devices 50, 1214 (2003).

${ }^{21}$ C. Oliviera, M. Massi, S. G. Santos, C. Otani, H. S. Maciel, and R. D. Mansano, Diam. Relat. Mater. 10, 1317 (2001).

${ }^{22}$ M. S. Lee, S. Wu, S. B. Jhong, K. H. Chen, and K. T. Liu, J. Nanomater. 2014, 250439.

${ }^{23}$ R. S. Muller and T. I. Kamins, Device Electronics for Integrated Circuits, 2nd ed. (Wiley, New York, 1986).

${ }^{24}$ N. B. Hassine, D. Mercier, P. Renaux, G. Parat, S. Basrour, P. Waltz, C. Chappaz, P. Ancey, and S. Blonkowski, J. Appl. Phys. 105, 044111 (2009).

${ }^{25}$ C. I. Wu and A. Kahn, Appl. Phys. Lett. 74, 546 (1999).

${ }^{26}$ D. W. Jenkins and J. D. Dow, Phys. Rev. B 39, 3317 (1989).

${ }^{27}$ V. Ligatchev, Rusli, and Z. Pan, Appl. Phys. Lett. 87, 242903 (2005). 